\title{
A randomised trial of lung sealant versus medical therapy for advanced emphysema
}

\author{
Carolyn E. Come, Mordechai R. Kramer, Mark T. Dransfield, \\ Muhanned Abu-Hijleh, David Berkowitz, Michela Bezzi, Surya P. Bhatt, \\ Michael B. Boyd, Enrique Cases, Alexander C. Chen, Christopher B. Cooper, \\ Javier Flandes, Thomas Gildea, Mark Gotfried, D. Kyle Hogarth, \\ Kumaran Kolandaivelu, William Leeds, Timothy Liesching, Nathaniel Marchetti, \\ Charles Marquette, Richard A. Mularski, Victor M. Pinto-Plata, \\ Michael A. Pritchett, Samaan Rafeq, Edmundo R. Rubio, Dirk-Jan Slebos, \\ Grigoris Stratakos, Alexander Sy, Larry W. Tsai, Momen Wahidi, John Walsh, \\ J. Michael Wells, Patrick E. Whitten, Roger Yusen, Javier J. Zulueta, \\ Gerard J. Criner and George R. Washko
}

Affiliations: For a full list of the authors' affiliations please see the Acknowledgements.

Correspondence: Carolyn E. Come, Pulmonary and Critical Care Division, Department of Medicine, Brigham and Women's Hospital, 75 Francis Street, PBB-Clinics 3, Boston, MA 02115 USA. E-mail: ccomedapartners.org

ABSTRACT Uncontrolled pilot studies demonstrated promising results of endoscopic lung volume reduction using emphysematous lung sealant (ELS) in patients with advanced, upper lobe predominant emphysema. We aimed to evaluate the safety and efficacy of ELS in a randomised controlled setting.

Patients were randomised to ELS plus medical treatment or medical treatment alone. Despite early termination for business reasons and inability to assess the primary 12-month end-point, 95 out of 300 patients were successfully randomised, providing sufficient data for 3- and 6-month analysis.

57 patients ( 34 treatment and 23 control) had efficacy results at 3 months; 34 (21 treatment and 13 control) at 6 months. In the treatment group, 3-month lung function, dyspnoea, and quality of life improved significantly from baseline when compared to control. Improvements persisted at 6 months with $>50 \%$ of treated patients experiencing clinically important improvements, including some whose lung function improved by $>100 \%$. $44 \%$ of treated patients experienced adverse events requiring hospitalisation (2.5-fold more than control, $\mathrm{p}=0.01$ ), with two deaths in the treated cohort. Treatment responders tended to be those experiencing respiratory adverse events.

Despite early termination, results show that minimally invasive ELS may be efficacious, yet significant risks (probably inflammatory) limit its current utility.

@ERSpublications

Emphysematous lung sealant therapy is highly efficacious in some patients, but benefits bring significant risks http://ow.ly/JJ2vg

Editorial comment in: Eur Respir J 2015; 46: 593-595 [DOI: 10.1183/09031936.00065715]

This article has supplementary material available from erj.ersjournals.com

Received: Nov 062014 | Accepted after revision: Feb 232015 | First published online: April 022015

Clinical trial: This trial is registered at clinicaltrials.gov with identifier number NCT01449292.

Support statement: Aeris Therapeutics funded the study. C.E. Come is supported by National Institutes of Health (NIH)/National Heart, Lung, and Blood Institute (K23HL114735). K. Kolandaivelu is supported by an American Heart Association FTF award (12FTF12080241). J.M. Wells is supported by NIH (K08HL1123940).

Conflict of interest: Disclosures can be found alongside the online version of this article at erj.ersjournals.com

Copyright $\odot$ ERS 2015 


\section{Introduction}

Lung volume reduction surgery (LVRS) improves quality of life, dyspnoea, lung function and exercise capacity, as well as mortality, in patients with severe upper lobe predominant emphysema and reduced exercise capacity $[1,2]$. Although it is effective, LVRS involves major surgery in patients with limited respiratory reserve and is associated with substantial morbidity and mortality. In the National Emphysema Treatment Trial (NETT), non-high-risk LVRS patients had a 90-day mortality of 5.5\%; 59\% experienced serious complications [3]. While risk may have declined with refinements in technique, LVRS remains rarely performed [4], and efforts are ongoing to develop less invasive endoscopic approaches capable of achieving similar benefits [5-16]. Although some techniques have proven safer than LVRS, they have also been less efficacious, with insufficient risk-benefit data in randomised clinical trials to obtain US Food and Drug Administration (FDA) approval [5, 7, 13, 15].

The emphysematous lung sealant system (ELS) (AeriSeal System; Aeris Therapeutics, Inc. Woburn, MA, USA) is an endoscopic lung volume reduction (ELVR) technique that employs a synthetic polymer to irreversibly block small airways and collateral channels, promoting atelectasis, remodelling and scar formation, thereby reducing lung hyperinflation. By sealing lung tissue at the alveolar level, this approach addresses the problem of collateral ventilation, a limitation of ELVR approaches employing mechanical devices to collapse the lung by blocking proximal airways [17]. In open-label pilot studies, ELS durably reduced lung volume, improving lung function and quality of life with an acceptable safety profile $[10,18,19]$.

Based on these promising findings, the AeriSeal System for Hyperinflation Reduction in Emphysema (ASPIRE) study was initiated. ASPIRE was an open-label, prospective, multicentre, randomised controlled trial comparing ELS treatment plus optimal medical therapy to optimal medical therapy alone in patients with advanced, upper lobe predominant emphysema. The study was terminated prematurely for business-related reasons after 95 out of 300 planned patients were randomised. However, accumulated data provide insight into the feasibility of using sealant therapy targeting the alveolar compartment in this high-risk population. Our results demonstrate that the procedure is technically simple, length of hospital stay generally brief, and surgical-level efficacy potentially achievable. Yet significant short-term side-effects associated with acute inflammation and infection risk may limit its potential utility in present form. Parts of this article have been reported in abstract form [20].

\section{Methods}

Patients

Between September 2012 and November 2013, 383 patients were screened at 37 centres; 241 were excluded and 47 were undergoing screening at study termination. 95 patients were randomised to ELS plus optimal medical therapy or optimal medical therapy alone [21]. Eligibility criteria included age $\geqslant 40$ years; former smoking (nonsmoking $\geqslant 4$ months) with $\geqslant 20$ pack-year history; upper lobe predominant emphysema on computed tomography (CT) (performed visually by site investigator and confirmed by study sponsor physicians); severe airflow obstruction with post-bronchodilator forced expiratory volume in $1 \mathrm{~s}$ (FEV1) $<50 \%$ predicted; hyperinflation, with total lung capacity $>100 \%$ pred and residual volume $>150 \%$ pred; single breath diffusing capacity of the lung for carbon monoxide $20-60 \%$ pred $[19,22]$; dyspnoea despite guideline-optimised medical therapy; post-rehabilitation 6-min walking distance (6MWD) $\geqslant 150 \mathrm{~m}$ [23]; carbon dioxide tension $<65 \mathrm{mmHg}$; and oxygen saturation $\geqslant 90 \%$ on $\leqslant 4 \mathrm{~L} \cdot \mathrm{min}^{-1}$ supplemental oxygen at rest. Exclusion criteria included prior lung volume reduction or lobectomy; clinically significant asthma or bronchiectasis; frequent severe chronic obstructive pulmonary disease (COPD) exacerbations; $\alpha_{1}$-antitrypsin deficiency; imaging consistent with active pulmonary infection, significant interstitial lung disease or pleural disease, giant bullous disease (predominant bulla $>10 \mathrm{~cm}$ in diameter) or a new lung nodule $>1 \mathrm{~cm}$ in diameter; active malignancy; HIV; recent myocardial infarction or stroke; pulmonary hypertension; impaired left ventricular function (ejection fraction $<45 \%$ ); body mass index $<15 \mathrm{~kg} \cdot \mathrm{m}^{-2}$ or $>35 \mathrm{~kg} \cdot \mathrm{m}^{-2}$; pregnancy/breastfeeding; significant abnormality in serum chemistries, complete blood count or coagulation indices; use of systemic corticosteroids at a daily dose greater than prednisone $20 \mathrm{mg}$ (or equivalent immunosuppressive agents); or use of anticoagulant medication.

\section{Study design}

The planned study schema is presented in figure 1. Prior to participation, subjects provided written informed consent. Patients underwent screening, including medical history, physical examination, blood work, pulmonary function tests, 6MWD, chest CT, electrocardiogram, echocardiogram and questionnaires. Those meeting the study criteria underwent pre-randomisation pulmonary rehabilitation, after which they returned for baseline evaluation. Eligible patients were randomised in a 3:2 ratio (treatment:control) to ensure adequate statistical power if there was significant drop-out in the control arm; the randomisation sequence was computer-generated in blocks of five, stratified by site. As per the FDA mandate, a sham procedure was not 
used, given the risks to control patients without potential benefit. Thus, patients and bronchoscopists were aware of treatment allocation; those conducting pulmonary function tests, 6MWD and questionnaires were blinded. After ELS (treatment group) or randomisation (control group), follow-up visits were planned at 3, 6, 9 and 12 months, followed by annual visits up to 5 years for treated patients. Control patients would be eligible for treatment after 12 months if they continued to meet inclusion/exclusion criteria and treatment was deemed safe. Patients were required to continue pulmonary rehabilitation post-randomisation. All patients, regardless of treatment allocation, continued optimal medical therapy, under the direction of their local physicians or study physicians. As the study was terminated prematurely, most randomised patients only underwent 3- or 6-month follow-up visits. The full protocol is provided in the online supplementary material.

\section{Procedure}

ELS was performed under moderate sedation or general anaesthesia. Prior to intervention, patients received the first doses of a 7-day steroid taper and prophylactic antibiotic course to reduce the

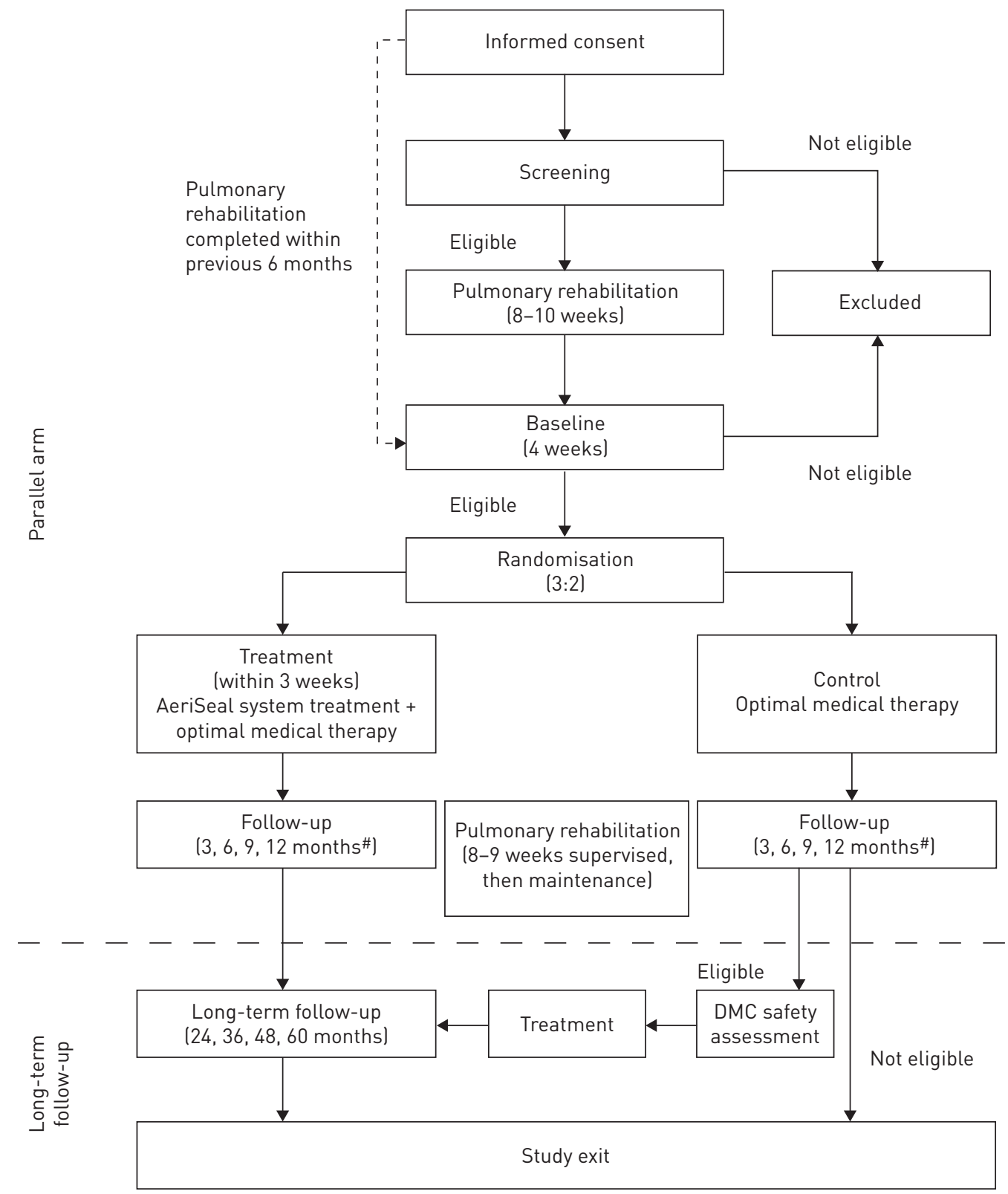

FIGURE 1 AeriSeal System for Hyperinflation Reduction in Emphysema study schema. DMC: data monitoring committee. ${ }^{\#}$ : telephone follow-up at 1,2 and 3 weeks and $1,2,4,5,7,8,10$ and 11 months. 
post-treatment acute inflammatory response (PAIR: fever, dyspnoea, cough, chest pain and/or elevated inflammatory markers) observed in pilot studies [19]. Patients also received prophylactic nonsteroidal anti-inflammatory drugs for 3 days post-treatment, and stress-ulcer prophylaxis. Target treatment sites, the most severely damaged upper lobe segments, were identified by site investigators based on CT review and confirmed by study sponsor physicians. Based on pilot studies, two upper lobe sub-segments in each lung were treated; procedures were performed in a single session (online supplementary material) [10]. Post-treatment, patients were monitored in hospital for $\geqslant 1$ night.

\section{Outcome measures}

Assessment of primary and secondary efficacy end-points, intended at 12-month follow-up, was available only at 3 and 6 months. The planned primary efficacy end-point was mean percentage change in post-bronchodilator FEV1 from baseline to 12 months. Secondary efficacy end-points included proportion of patients achieving minimal clinically important differences (MCID) in FEV1 (MCID $\geqslant 100 \mathrm{~mL}$ and 12\%); [24] dyspnoea quantified by the modified Medical Research Council dyspnoea scale (mMRC) (0-4, a higher score indicating more severe dyspnoea and MCID $\geqslant 1 \mathrm{U}$ decrease) [25]; and disease-specific quality of life measured by St George's Respiratory Questionnaire (SGRQ) (0-100: a higher score indicating worse quality of life and MCID $\geqslant 4$ U decrease) [26]; as well as changes in 6MWD [27] and upper lobe volume (measured by quantitative CT) at 12 months. Adverse events were recorded, focusing on serious adverse events, including deaths and those requiring hospitalisation.

\section{Study administration and oversight}

The study was sponsored by Aeris Therapeutics, Inc. The protocol was developed by the sponsor in collaboration with the Center for Devices and Radiological Health of the FDA and an independent data monitoring committee (DMC), comprising three physicians and a statistician. The protocol was approved by the institutional review boards and ethics committees of participating centres. The DMC assisted with developing safety-related stopping rules, reviewed safety data bi-annually and evaluated deaths. Recommendations were forwarded to the sponsor. An independent, blinded medical assessment committee reviewed and adjudicated all serious adverse events and a pre-defined subset of respiratory-related adverse events (i.e. pneumonia, pneumothorax, respiratory failure, haemoptysis and COPD exacerbation).

\section{Study termination}

The study was stopped prematurely due to lack of company financing. While the adverse event rate was higher than anticipated, the DMC did not recommend trial termination. At termination, a group of participating physicians obtained data collected per protocol from the sponsor, providing the content for this article.

\section{Statistical analysis}

Given the early termination of the trial, descriptive statistics for subjects at baseline and 3 and 6 months post-randomisation are presented. Efficacy analyses using interim assessments were performed according to intention-to-treat in subjects with available data. Safety analyses used the as-treated population. As sample sizes were limited, continuous variables are presented as median (interquartile range) and dichotomous variables as $\mathrm{n}(\%)$, unless otherwise stated. Non-parametric tests were used for between-group comparisons and p-values reported.

\section{Results}

Study patients

61 patients were randomised to ELS; 34 to control treatment (fig. 2). Study groups were well matched at baseline (table 1), except control patients had more upper lobe predominant emphysema on CT. 59 of those randomised to treatment received ELS, two did not. One withdrew prior to treatment; the study was terminated before treating the other. At study termination, results were available for 57 patients (34 in the treatment group and 23 in the control group) at 3 months and 34 patients ( 21 in the treatment group and 13 in the control group) at 6 months. One control patient withdrew on day 81 to pursue alternative therapy.

\section{Procedural details}

All patients were successfully treated at two sub-segments in each upper lobe. 28 procedures were performed under general anaesthesia and 31 under conscious (moderate) sedation. Mean $\pm \mathrm{SD}$ duration of ELS was $23 \pm 8 \mathrm{~min}$ (median $22 \mathrm{~min}$, minimum $8 \mathrm{~min}$ and maximum $47 \mathrm{~min}$ ). Patients spent mean $\pm \mathrm{SD}$ $5.3 \pm 17.9$ days (median 1 day, minimum 1 day per protocol and maximum 120 days) in the hospital post-procedure. ELS was well tolerated. No adverse events occurred during the technical procedure, and 


\begin{tabular}{|c|c|c|c|}
\hline & Treatment & Control & p-value \\
\hline Subjects $n$ & 61 & 34 & \\
\hline \multicolumn{4}{|l|}{ Demographics } \\
\hline Age years & $65(58-69)$ & $64(58-69)$ & 0.69 \\
\hline Male & $35(57)$ & $21(62)$ & 0.68 \\
\hline White $^{\#}$ & $58(95)$ & 33 (97) & 0.99 \\
\hline $\mathrm{BMI} \mathrm{kg} \cdot \mathrm{m}^{-2}$ & $25.2(21.9-27.9)$ & $25.7(21.4-28.3)$ & 0.90 \\
\hline Smoking pack-years & $50(37-77)$ & $50(39-65)$ & 0.97 \\
\hline Supplemental oxygen & $41(67)$ & $25(74)$ & 0.52 \\
\hline \multicolumn{4}{|l|}{ Lung function" } \\
\hline FEV $1 \mathrm{~L}$ & $0.78(0.65-1.02)$ & $0.90(0.70-1.06)$ & 0.274 \\
\hline $\mathrm{FEV}_{1} \%$ pred & $29(23-35)$ & $30(27-38)$ & 0.132 \\
\hline FVC L & $2.39(1.97-3.02)$ & $2.52(2.02-3.18)$ & 0.630 \\
\hline TLC L & $7.42(6.18-8.58)$ & $6.99(6.16-7.94)$ & 0.338 \\
\hline TLC \% pred & $124(115-139)$ & $120(108-133)$ & 0.088 \\
\hline RV L & $4.35(3.73-5.19)$ & $4.04(3.70-4.53)$ & 0.159 \\
\hline RV \% pred & $200(168-231)$ & $179(168-215)$ & 0.140 \\
\hline$D \mathrm{Lco} \mathrm{mL} \cdot \mathrm{min}^{-1} \cdot \mathrm{mmHg}^{-1}$ & $7.90(6.13-10.36)$ & $8.06(7.18-10.91)$ & 0.378 \\
\hline DLco \% pred & $33(26-39)$ & $36(28-46)$ & 0.235 \\
\hline \multicolumn{4}{|l|}{ Arterial blood gases ${ }^{+}$} \\
\hline $\mathrm{PaO}_{2} \mathrm{mmHg}$ & $70(64-78)$ & $71(63-78)$ & 0.917 \\
\hline $\mathrm{PaCO}_{2} \mathrm{mmHg}$ & $41(37-45)$ & $41(37-44)$ & 0.837 \\
\hline \multicolumn{4}{|l|}{ Exercise performance } \\
\hline $\begin{array}{l}\text { 6MWD m } \\
\mathrm{CT}^{\S}\end{array}$ & $313(236-363)$ & $293(247-420)$ & 0.277 \\
\hline Emphysema \% & $34.5(27.8-44.4)$ & $30.0(20.6-39.3)$ & 0.052 \\
\hline Heterogeneity index & $1.45(1.27-1.72)$ & $1.60(1.40-2.06)$ & 0.035 \\
\hline \multicolumn{4}{|l|}{ Questionnaires } \\
\hline mMRC U & $3.0(2.0-3.0)$ & $2.0(2.0-3.0)$ & 0.452 \\
\hline SGRQ U & $54(46-65)$ & $58(45-74)$ & 0.508 \\
\hline \multicolumn{4}{|l|}{ Medication use } \\
\hline Short-acting bronchodilator & $51(84)$ & $26(77)$ & 0.395 \\
\hline Long-acting bronchodilator ${ }^{f}$ & $48(79)$ & $23(68)$ & 0.235 \\
\hline Inhaled corticosteroid & $47(77)$ & $21(62)$ & 0.113 \\
\hline Oral corticosteroid & $9(15)$ & $3(9)$ & 0.623 \\
\hline
\end{tabular}

Data are presented as median (interquartile range) or $\mathrm{n}(\%)$, unless otherwise stated. BMI: body mass index; FEV1: forced expiratory volume in $1 \mathrm{~s} ; \%$ pred: \% predicted; FVC: forced vital capacity; TLC: total lung capacity; RV: residual volume; $D\left\llcorner c 0\right.$ : single breath diffusing capacity of the lung for carbon monoxide; $P_{a O_{2}}$ : arterial oxygen tension; $\mathrm{PaCO}_{2}$ : arterial carbon dioxide tension; 6MWD: 6-min walking distance; $\mathrm{CT}$ : computed tomography; mMRC: modified Medical Research Council dyspnoea scale; SGRQ: St George's Respiratory Questionnaire. \#: race was self-reported. "?: all lung function measures were obtained post-bronchodilator. ${ }^{+}$: arterial blood gas measurements were performed while patients were breathing ambient air. ${ }^{\S}$ : per cent emphysema was defined as the percentage of total lung tissue with a density <-950 HU. Heterogeneity index $=[\%$ voxels in the right upper lobe+left upper lobe $<-910 \mathrm{HU}] /[\%$ voxels in the right lower lobe+left lower lobe $<-910 \mathrm{HU}$ [ [10], with higher indices indicating more pronounced upper lobe predominant emphysema. $f$ : long-acting bronchodilator includes long-acting $\beta$-agonists, long-acting anticholinergics or both.

there were no procedure-related deaths. In general, patients were hospitalised longer than the required observation period for respiratory insufficiency in the setting of anticipated PAIR.

\section{Efficacy outcomes}

At 3 months, FEV1 improvement was $11.4 \%(2.0-32.0 \%)$ and $110 \mathrm{~mL}(18-211 \mathrm{~mL})$ in the treatment group versus $-2.1 \%(-4.9-9.0 \%)$ and $-20 \mathrm{~mL}(-48-60 \mathrm{~mL})$ in controls $(\mathrm{p}=0.0037)$. There were also improvements in health-related quality of life (change in SGRQ -11 U (-18--1 U) versus $-4 \mathrm{U}(-6-3 \mathrm{U}), \mathrm{p}=0.026)$ and dyspnoea (change in mMRC $-1.0 \mathrm{U}(-2.0-0 \mathrm{U})$ versus $0 \mathrm{U}(-0.8-0.8 \mathrm{U}), \mathrm{p}=0.005)$ in the treatment group compared with controls.

Improved lung function, exercise capacity and health-related quality of life were observed at 6 months in treatment versus control groups. Change in FEV1 change was $18.9 \%(-0.7-41.9 \%)$ and $100 \mathrm{~mL}(0-370 \mathrm{~mL})$ in the treatment group versus $1.3 \%(-8.2-12.9 \%)$ and $10 \mathrm{~mL}(-90-100 \mathrm{~mL})$ in controls $(\mathrm{p}=0.043)$. Treated patients 


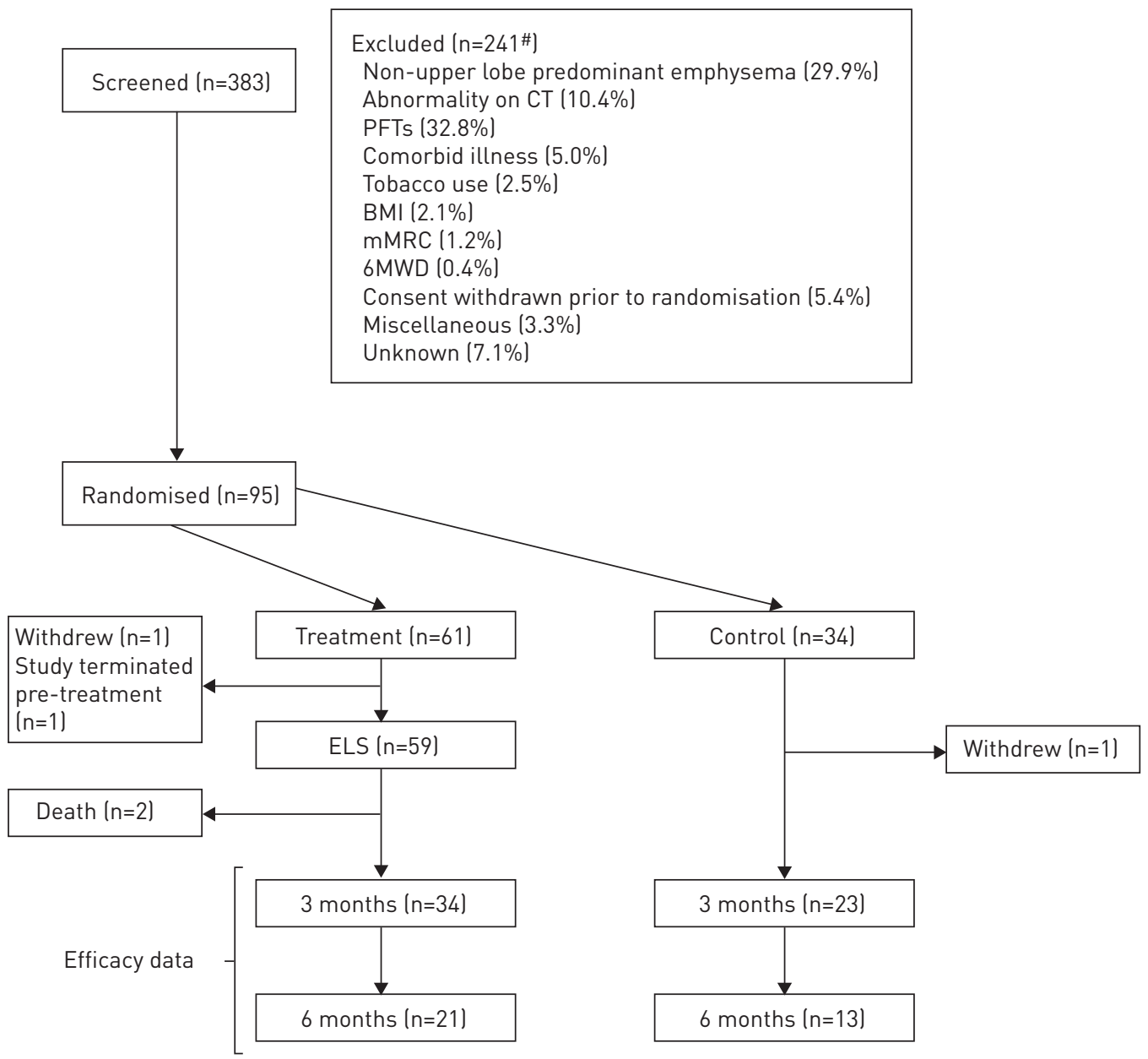

FIGURE 2 Consort diagram summarising enrolment, allocation, and follow-up. CT: computed tomography; PFTs: pulmonary function tests; BMI: body mass index; mMRC: modified Medical Research Council dyspnoea scale; 6MWD: 6-min walking distance; ELS: emphysematous lung sealant system. " : 47 patients were undergoing screening at the time of study termination.

had improvements in 6MWD compared with controls $(31.0 \mathrm{~m}(0-41.3 \mathrm{~m})$ versus $-22.0 \mathrm{~m}(-41.3-9.3 \mathrm{~m})$, $\mathrm{p}=0.019$; data not collected at 3 months) as well as in SGRQ (-12 U (-22--5 U) and $-3 \mathrm{U}(-5-1 \mathrm{U})$ in treatment and control groups, respectively; $\mathrm{p}=0.0072)$. There was no difference in dyspnoea between groups at 6 months (mMRC $-1.0 \mathrm{U}(-1.0-0 \mathrm{U})$ in treated patients versus $0 \mathrm{U}(-1.0-0 \mathrm{U})$ controls; $\mathrm{p}=0.57)$. FEV1 and SGRQ at baseline, 3 months and 6 months for each patient with 6-month follow-up data are plotted in online figure S1. The proportions of patients in each group achieving MCID in measured variables at 3 and 6 months are presented in table 2. Figure 3 depicts changes in $\mathrm{FEV}_{1}$ at 6 months for individual patients. $>50 \%$ of treated patients had a MCID in FEV1 at 6 months versus $15 \%$ of control patients. Baseline and 6-month CT images for one treated patient demonstrate radiographic evidence of upper lobe deflation and lower lobe inflation with corresponding improvement in airflow obstruction (fig. 4).

\section{Adverse events}

Two deaths occurred in the treatment group, both beyond the immediate peri-procedure period. One was due to a myocardial infarction 55 days after treatment in a patient treated for a COPD exacerbation/ pneumonia $\sim 40$ days following intervention. The second death occurred in a patient who developed pneumonia at a treatment site and died of sepsis 65 days post-procedure. There were no deaths in the control group ( $p$-value for mortality difference 0.80 ). Three patients in the treatment group experienced four episodes of respiratory failure requiring invasive mechanical ventilation. Two of these patients required tracheostomy, but were eventually decannulated. There were no episodes of respiratory failure in the controls. $44 \%$ of treated patients and $18 \%$ of controls experienced adverse events requiring hospitalisation $(\mathrm{p}=0.0098)$ at any time during the study. The majority of events were respiratory $(77 \%$ and $89 \%$ of events in 
the treatment and control groups, respectively). Serious adverse events are summarised in table 3. Figure 5 shows examples of treatment-associated pulmonary complications, including pulmonary infiltrate, pleural effusion and possible lung abscess. For each patient with 6 months follow-up, the number of adverse events

\section{TABLE 2 Proportion of patients achieving minimally clinically important differences in} measured variables

\begin{tabular}{|c|c|c|c|c|c|c|}
\hline & \multicolumn{3}{|c|}{3 months } & \multicolumn{3}{|c|}{6 months } \\
\hline & Treatment & Control & p-value & Treatment & Control & p-value \\
\hline Subjects $n$ & 34 & 23 & & 21 & 13 & \\
\hline $\mathrm{FEV}_{1}{ }^{\#}$ & 47.1 & 8.7 & 0.001 & 52.4 & 15.4 & 0.068 \\
\hline SGRQ" & 58.8 & 47.8 & 0.414 & 76.2 & 46.2 & 0.159 \\
\hline $\mathrm{mMRC}^{+}$ & 55.9 & 26.1 & 0.026 & 52.4 & 38.5 & 0.664 \\
\hline $6 M W D^{\S}$ & NA & NA & NA & 52.4 & 0 & 0.0025 \\
\hline
\end{tabular}

Data are presented as \%, unless otherwise stated. FEV1: forced expiratory volume in $1 \mathrm{~s} ; \mathrm{SGRQ}$ : St George's Respiratory Questionnaire; mMRC: modified Medical Research Council dyspnoea scale; 6MWD: 6-min walking distance; NA: not available. " $:$ MCID $\geqslant 12 \%$ and $100 \mathrm{~mL}$ [24] increase in post-bronchodilator FEV1 from baseline; ${ }^{\text {१: }}$ MCID $\geqslant 4$ unit decrease from baseline [26]; ${ }^{+}$: MCID $\geqslant 1$ unit decrease from baseline [25]; ${ }^{\S}$ : MCID $26 \mathrm{~m}$ increase from baseline [27].
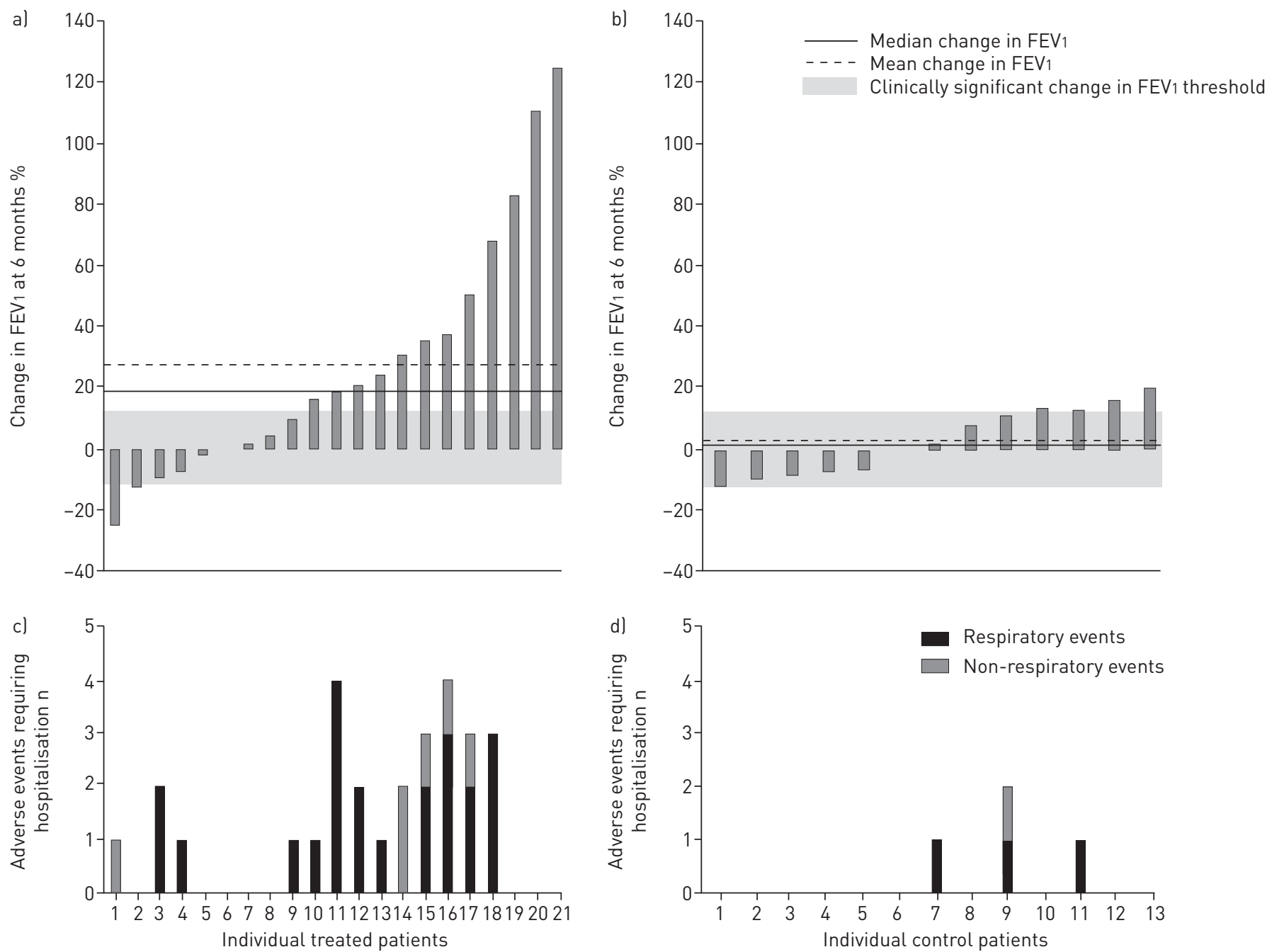

FIGURE 3 Percentage change in forced expiratory volume in $1 \mathrm{~s}$ (FEV1) from baseline to 6-month follow-up for a) treated patients and b) control patients. Each bar represents an individual patient. The regions outside the shaded zone indicate clinically significant changes in FEV 1 . Adverse events requiring hospitalisation for the corresponding individual c) treated patients and d) control patients. 
requiring hospitalisation is presented in figure 3, concurrent with change in FEV1. In treated patients, there was a trend toward FEV1 responders (those with a MCID in FEV1) being more likely than nonresponders to have had respiratory adverse events ( $67 \%$ versus $27 \%, \mathrm{p}=0.139)$.
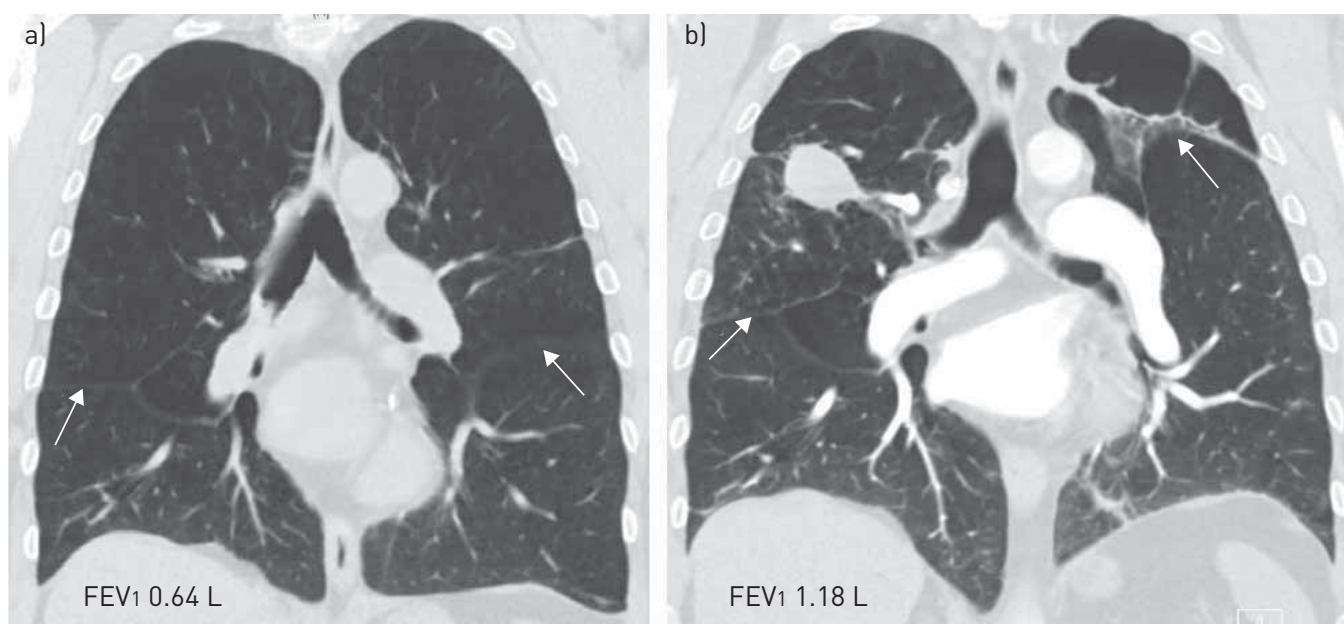

FIGURE 4 Coronal images at equivalent anatomic locations from chest computed tomographic (CT) scans of the same patient at various time points, with corresponding forced expiratory volume in $1 \mathrm{~s}$ (FEV1). The patient had a complicated post-treatment course with repeated admissions for fevers and pneumonia versus pneumonitis. $\sim 6$ months after treatment (after termination of the study) the patient was found to have a pulmonary embolus in the left upper lobar artery extending into segmental and subsegmental arteries. Given the location of the clot within collapsed/treated areas, there was suspicion of in situ thrombosis. The patient was treated with anticoagulant therapy. a) Baseline chest $\mathrm{CT}$; b) clinically-acquired chest CT taken almost 6 months post-treatment demonstrates a treatment site in the right upper lobe. Compared with the baseline CT, the diaphragms and fissures (arrows) are now elevated, consistent with deflation. The patient's FEV1 improved $84 \%$ from baseline (follow-up spirometry obtained outside the study).

TABLE 3 Serious adverse events

\begin{tabular}{|c|c|c|c|c|c|c|c|c|}
\hline & \multicolumn{2}{|c|}{$\begin{array}{c}0-30 \text { days } \\
\text { Patients (events) }\end{array}$} & \multicolumn{2}{|c|}{$\begin{array}{c}\text { 31-60 days } \\
\text { Patients (events) }\end{array}$} & \multicolumn{2}{|c|}{$\begin{array}{c}61-90 \text { days } \\
\text { Patients (events) }\end{array}$} & \multicolumn{2}{|c|}{$\begin{array}{c}>90 \text { days } \\
\text { Patients (events) }\end{array}$} \\
\hline & Treatment & Control & Treatment & Control & Treatment & Control & Treatment & Control \\
\hline Death & & & $1(1)$ & & $1(1)$ & & & \\
\hline Respiratory failure ${ }^{\#}$ & $3(3)$ & & $1(1)$ & & & & & \\
\hline Pneumonia & 2 (3) & & $6(7)$ & & $4(5)$ & & $3(3)$ & $2(2)$ \\
\hline COPD exacerbation & $5(5)$ & 2 (2) & 1 (1) & $1(2)$ & $1(1)$ & & $4(6)$ & $1(1)$ \\
\hline PAIR & $4(5)$ & & 1 (1) & & & & & \\
\hline Pneumothorax & 1 (2) & & & & & & & \\
\hline Lung cavity & & & & & & & $1(1)$ & \\
\hline Lung mass & & & & & & & & $1(1)$ \\
\hline Dyspnoea & $1(1)$ & & & & & & & \\
\hline Myocardial infarction & & & $1(1)$ & & & & & \\
\hline Chest pain & & & $1(1)$ & & & & & \\
\hline Tachyarrhythmia & & & & & & & $1(1)$ & $1(1)$ \\
\hline Sepsis & & & $1(1)$ & & $1(1)$ & & & \\
\hline Fever & & & $1(1)$ & & & & & \\
\hline Acute kidney injury & & & $1(1)$ & & & & & \\
\hline Urinary tract infection & & & & & $1(1)$ & & $1(1)$ & \\
\hline Ileus & & & $1(1)$ & & & & & \\
\hline Inguinal hernia & & & & & & & $1(1)$ & \\
\hline Depression & & & $1(1)$ & & & & & \\
\hline
\end{tabular}

Data are presented as $\mathrm{n}(\mathrm{n})$. Respiratory events are highlighted in grey. COPD: chronic obstructive pulmonary disease; PAIR: post-treatment acute inflammatory response (fever, dyspnoea, cough, chest pain and/or elevated inflammatory markers). \#: respiratory failure was considered an outcome rather than an event, so was not included in the composite of respiratory events. 

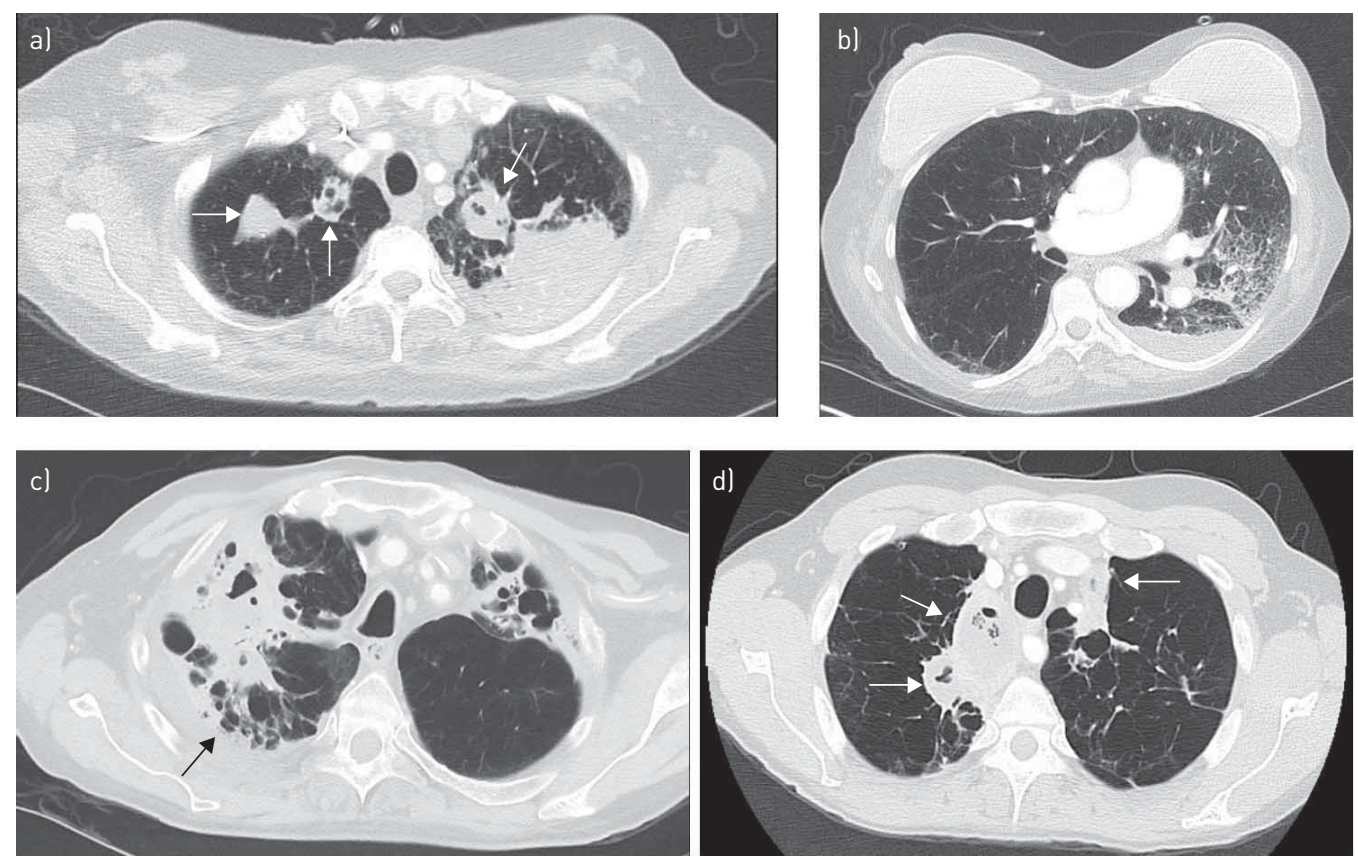

FIGURE 5 Axial images from chest computed tomography (CT) scans (performed for clinical indications) of patients treated with the emphysematous lung sealant system, displaying the appearance of the lungs and potential complications post-treatment. a, b) CT obtained 66 days after treatment when patient 1 presented with dyspnoea and abdominal pain. The patient was treated with broad-spectrum antibiotics for pneumonia. A thorough abdominal work-up was negative. Steroids were added to reduce potential systemic inflammation as a result of her treatment (C-reactive protein $66.3 \mathrm{mg} \cdot \mathrm{L}^{-1}$ ); her symptoms improved rapidly. a) Bilateral apical opacities consistent with treatment sites (arrows), as well as dense consolidation in the posterior left upper lobe (beyond expected treatment site). b) Ground glass opacification in the untreated left lower lobe and a small pleural effusion. c) CT scan obtained 70 days after treatment when patient 2 presented with persistent shortness of breath and fevers despite treatment with broad-spectrum antibiotics. CT shows bilateral upper lobe consolidation and a right-sided pleural effusion (arrow; transudative). The patient's condition improved upon treatment with steroids. d) CT obtained 23 days after treatment when patient 3 presented with fever, lethargy, and rust-colored sputum. CT demonstrates consolidation and complex air-fluid collections bilaterally, which may indicate lung abscesses rather than post-treatment changes (arrows). The patient improved upon treatment with broad-spectrum antibiotics.

\section{Discussion}

At termination of this randomised controlled trial, ELS therapy compared to medical treatment produced encouraging physiological, functional, and quality-of-life benefits for up to 6 months in patients with severe upper lobe predominant emphysema. While treatment was associated with frequent adverse events and the time for primary end-point data collection was not reached, accrued data suggest that this novel approach, utilising a polymer sealant that flows into the alveolar compartment and collapses the hyperinflated lung is procedurally well-tolerated and can be highly effective in this high-risk group. $>50 \%$ of patients randomised to treatment experienced MCID in FEV1, SGRQ, mMRC and 6MWD at 6 months.

These findings are consistent with ELS pilot studies [10, 18, 19]. In 20 patients undergoing bilateral upper lobe treatment with ELS, KRAMER et al. reported significant improvements in FEV1, dyspnoea and hyperinflation at 12 months, confirming the efficacy of this approach [10]. Long-term follow-up of this cohort for 2 years revealed a trend toward improvement in FEV1 (mean \pm SD change $14.5 \pm 33.1 \%, \mathrm{p}=0.058$ ) with eight out of 16 patients achieving a MCID in FEV1, suggesting durability of this approach [28]. The current randomised study extends this work by adding a critical control arm.

In our limited data set, efficacy results with ELS approached those of surgical lung volume reduction, although overall adverse events were also prominent. In NETT, the mean percentage change in FEV 1 from baseline to 6 months for non-high risk LVRS patients was $28.5 \pm 33.6 \%$ compared with $27.5 \pm 40.1 \%$ in ASPIRE (fig. 3) [1]. Improvements in respiratory-specific health-related quality of life were also comparable (mean absolute change in SGRQ was $-11 \pm 15 \mathrm{U}$ in NETT versus $-12 \pm 15 \mathrm{U}$ in this study at 6 months) [1]. 90-day all-cause mortality after LVRS was 5.5\% (non-high-risk patients) [3] and 3.4\% post-ELS therapy. While the combined incidence of post-treatment respiratory failure and pneumonia in the first 30 days following ELS was lower than following surgery (10.2\% versus 54\% in NETT) [3], in ASPIRE, adverse events 
continued through later follow-up (overall incidence of post-ELS respiratory failure and pneumonia 37\%), potentially indicative of distinct pathological mechanisms between surgical and sealant-based approaches.

Ongoing adverse events following ELS may result from intense subacute inflammation arising from chemically-induced collapse and scarring of hyperinflated lung tissue. In a separate study of 29 patients treated with ELS, mean C-reactive protein (CRP) level 3 months post-treatment rose three-fold compared to baseline, indicative of significant inflammation. By 6 months, CRP dropped below baseline, remaining so at 1 year [29]. Given the premature termination of this study, the incidence of ELS-related adverse events after this potential "inflammatory window" remains unclear, although in the pilot study [28], no COPD exacerbations or pneumonia occurred after the first year.

ELS in its current form has clear limitations. Beyond adverse events, the therapy is irreversible, in contrast to other ELVR techniques. Still, the potential benefits of technological and procedural modifications, though speculative, merit consideration. For example, all patients received treatment at two upper lobe subsegments in a single session. While a staged procedure may have prolonged the inflammatory response, the intensity of the response may have been attenuated. It is possible that treating the most diseased side first, and potentially only, may have resulted in adequate efficacy with reduced risk. Changes in sealant formulation that reduce chemical irritation may also help achieve an ELS-like product with an improved safety profile. Moreover, the small number of participants with follow-up data precluded our ability to determine patient-specific characteristics predictive of those most likely to achieve benefit and those most likely to experience harm.

Such modifications may not positively alter the risk:benefit profile of ELS. Indeed, treatment responders in this study tended to have more respiratory adverse events. Considering data from available randomised controlled trials, it is possible that safer ELVR therapies may intrinsically be associated with reduced efficacy (i.e. endobronchial valves and airway bypass) $[5,13]$, while more effective treatments may have more severe side-effects $[9,11,16]$, even when the mechanism of action is not clearly linked with inflammation $[9,16]$. For example, while $>50 \%$ of patients treated with vapuor ablation or airway coil therapy experienced clinically important improvements in FEV1, SGRQ and 6MWD, the incidence of serious respiratory adverse events at 90 days was $57 \%$ and $39 \%$ in each study, respectively $[9,11]$. Even with endobronchial valves, which have generally proven less efficacious, greatest response (i.e. in cases with intact fissures and lobar occlusion) has been linked with higher risk (more pneumothoraces) [5, 30, 31]. Thus, changing the anatomical structure of the lung sufficiently to achieve clinical benefit may inherently incur higher risk, even if achieved through a less invasive, endoscopic approach and regardless of method or degree of inflammation.

Despite its early termination, this study provides controlled proof of concept of sealant-based ELVR. The procedure itself was associated with low morbidity and high technical success, yet efficacy was offset by significant cumulative adverse events. For patients with advanced emphysema and few options, these results are disappointing. At a time when access to lung transplantation for end-stage emphysema is becoming more restricted due to new organ allocation guidelines [32], and patients are often too sick to undergo surgery, minimally invasive therapies are desperately needed, yet remain unapproved in USA. If effective ELVR therapy is ultimately determined to have a high inherent risk, independent of technique, then such treatments may be restricted to only the healthiest, most functional patients with end-stage lung disease, leaving a significant residual unmet medical need. Alternatively, we may need to re-examine the acceptable balance of therapy-related side-effects against the potential for significant clinical benefit in end-stage emphysema.

\section{Acknowledgements}

The authors' affiliations are as follows. C. Come: Dept of Medicine, Division of Pulmonary and Critical Care, Brigham and Women's Hospital, Boston, MA, USA; M.R. Kramer: Pulmonary Institute, Rabin Medical Center, Petach Tikva, Israel; M.T. Dransfield: Dept of Medicine, Division of Pulmonary, Allergy \& Critical Care Medicine, University of Alabama at Birmingham, Birmingham, AL, USA; M. Abu-Hijleh: Dept of Medicine, UT Southwestern Medical Center Dallas, Dallas, TX, USA; D. Berkowitz: Dept of Medicine, Pulmonary Division, Emory University Hospital, Atlanta, GA, USA; M. Bezzi: Dept of Pneumonology, Azienda Ospedaliera Spedali Civili Brescia, Brescia, Italy; S.P. Bhatt: Dept of Medicine, Division of Pulmonary, Allergy \& Critical Care Medicine, University of Alabama at Birmingham, Birmingham, AL, USA; M.B. Boyd: Dept of Pulmonary Medicine, Sleep Medicine, Critical Care Medicine, Carilion Roanoke Memorial Hospital, Roanoke, VA, USA; E. Cases: Servicio de Neumología, Hospital Universitario y Politécnico La Fe, Valencia, Spain; A.C. Chen: Dept of Medicine, Division of Pulmonary and Critical Care Medicine, Washington University School of Medicine, St. Louis, MO, USA; C.B. Cooper: Dept of Medicine, Pulmonary Disease, David Geffen School of Medicine at UCLA, Los Angeles, CA, USA; J. Flandes: Neumología, Fundacion Jimenez Diaz Hospital, Madrid, Spain; T. Gildea: Pulmonary Medicine Dept, Cleveland Clinic, Cleveland, OH, USA; M. Gotfried: Pulmonary Associates, Phoenix, AZ, USA; D.K. Hogarth: Pulmonary and Critical Care Medicine, University of Chicago Medicine, Chicago, IL, USA; K. Kolandaivelu: Dept of Medicine, Division of Cardiology, Brigham and Women's Hospital, Boston, MA, USA; W. Leeds: Veritas Clinical Specialties, Topeka, KS, USA; T. Liesching: Pulmonary and Critical Care Medicine, Lahey Clinic, Burlington, MA, USA; N. Marchetti: Dept of Thoracic Medicine and Surgery, Temple University Hospital, Philadelphia, PA, USA; C. Marquette: Service de Pneumologie, Hôpital Pasteur Centre Hospitalier Universitaire de Nice, Nice, France; R.A. Mularski: Science Programs Dept, The Center for Health Research, Kaiser Permanente Northwest, Portland, OR, USA; V.M. Pinto-Plata: Dept of Medicine, Division of Pulmonary and Critical Care, Brigham and Women's Hospital, Boston, MA, USA; M.A. Pritchett: Pulmonary Medicine, Pinehurst Medical Center, Sanford, NC, 
USA; S. Rafeq: Division of Pulmonary and Critical Care and Sleep Medicine, St Elizabeth's Medical Center, Brighton, MA, USA; E.R. Rubio: Dept of Pulmonary Medicine, Sleep Medicine, Critical Care Medicine, Carilion Roanoke Memorial Hospital, Roanoke, VA, USA; D-J. Slebos: Dept of Pulmonary Diseases/Interventional Bronchoscopy, University Medical Center Groningen, Groningen, The Netherlands; G. Stratakos: Respiratory Medicine Dept, University of Athens "Sotiria" General Hospital, Athens, Greece; A. Sy: Dept of Medicine, Division of Pulmonary, Allergy, and Critical Care Medicine, Duke University Medical Center, Durham, NC, USA; L.W. Tsai: Dept of Medicine, Division of Pulmonary and Critical Care, Brigham and Women's Hospital, Boston, MA, USA; M. Wahidi: Dept of Medicine, Division of Pulmonary, Allergy, and Critical Care Medicine, Duke University Medical Center, Durham, NC, USA; J. Walsh: Pulmonary Disease, Presence St Joseph Medical Center, Joliet, IL, USA; J.M. Wells: Dept of Medicine, Division of Pulmonary, Allergy \& Critical Care Medicine, University of Alabama at Birmingham, Birmingham, AL, USA; P.E. Whitten: Pulmonary Disease, Illinois Lung Institute, Peoria, IL, USA; R. Yusen: Divisions of Pulmonary and Critical Care Medicine and General Medical Sciences, Washington University School of Medicine, St. Louis, MO, USA; J.J. Zulueta: Departmento de Neumología, Clinica Universidad de Navarra, Pamplona, Spain; G.J. Criner: Dept of Thoracic Medicine and Surgery, Lahey Clinic, Burlington, MA, USA; G.R. Washko: Dept of Medicine, Division of Pulmonary and Critical Care, Brigham and Women's Hospital, Boston, MA, USA.

\section{References}

1 Fishman A, Martinez F, Naunheim K, et al. A randomized trial comparing lung-volume-reduction surgery with medical therapy for severe emphysema. N Engl J Med 2003; 348: 2059-2073.

2 Naunheim KS, Wood DE, Mohsenifar Z, et al. Long-term follow-up of patients receiving lung-volume-reduction surgery versus medical therapy for severe emphysema by the National Emphysema Treatment Trial Research Group. Ann Thorac Surg 2006; 82: 431-443.

3 Naunheim KS, Wood DE, Krasna MJ, et al. Predictors of operative mortality and cardiopulmonary morbidity in the National Emphysema Treatment Trial. J Thorac Cardiovasc Surg 2006; 131: 43-53.

4 Akuthota P, Litmanovich D, Zutler M, et al. An evidence-based estimate on the size of the potential patient pool for lung volume reduction surgery. Ann Thorac Surg 2012; 94: 205-211.

5 Sciurba FC, Ernst A, Herth FJ, et al. A randomized study of endobronchial valves for advanced emphysema. N Engl J Med 2010; 363: 1233-1244.

6 Sterman DH, Mehta AC, Wood DE, et al. A multicenter pilot study of a bronchial valve for the treatment of severe emphysema. Respiration 2010; 79: 222-233.

7 Wood DE, Nader D, Elstad MR, et al. Bronchial valve treatment of emphysema: results and conclusions from a double-blind randomized trial. Am J Respir Crit Care Med 2012; 185: A5348.

8 Elstad MR, Mehta AC, Nader D, et al. Bronchial valve treatment of emphysema: procedure and device safety results from a double-blind randomized trial. Am J Respir Crit Care Med 2012; 185: A1112.

9 Shah PL, Zoumot Z, Singh S, et al. Endobronchial coils for the treatment of severe emphysema with hyperinflation (RESET): a randomised controlled trial. Lancet Respir Med 2013; 1: 233-240.

10 Kramer MR, Refaely Y, Maimon N, et al. Bilateral endoscopic sealant lung volume reduction therapy for advanced emphysema. Chest 2012; 142: 1111-1117.

11 Snell G, Herth FJ, Hopkins P, et al. Bronchoscopic thermal vapour ablation therapy in the management of heterogeneous emphysema. Eur Respir J 2012; 39: 1326-1333.

12 Herth FJ, Ernst A, Baker KM, et al. Characterization of outcomes 1 year after endoscopic thermal vapor ablation for patients with heterogeneous emphysema. Int J Chron Obstruct Pulmon Dis 2012; 7: 397-405.

13 Shah PL, Slebos DI, Cardoso PF, et al. Bronchoscopic lung-volume reduction with Exhale airway stents for emphysema (EASE trial): randomised, sham-controlled, multicentre trial. Lancet 2011; 378: 997-1005.

14 Ingenito EP, Wood DE, Utz JP. Bronchoscopic lung volume reduction in severe emphysema. Proc Am Thorac Soc 2008; 5: 454-460.

15 Ninane V, Geltner C, Bezzi M, et al. Multicentre European study for the treatment of advanced emphysema with bronchial valves. Eur Respir J 2012; 39: 1319-1325.

16 Slebos DJ, Klooster K, Ernst A, et al. Bronchoscopic lung volume reduction coil treatment of patients with severe heterogeneous emphysema. Chest 2012; 142: 574-582.

17 Fessler HE. Collateral ventilation, the bane of bronchoscopic volume reduction. Am J Respir Crit Care Med 2005; 171: 423-424.

18 Herth FJ, Gompelmann D, Stanzel F, et al. Treatment of advanced emphysema with emphysematous lung sealant (AeriSeal ${ }^{\circledR}$ ). Respiration 2011; 82: 36-45.

19 Herth FJ, Eberhardt R, Ingenito EP, et al. Assessment of a novel lung sealant for performing endoscopic volume reduction therapy in patients with advanced emphysema. Expert Rev Med Devices 2011; 8: 307-312.

20 Washko GR, Abu-Hijleh MA, Berkowitz D, et al. Results of the ASPIRE Endoscopic Lung Volume Reduction Trial at study termination. Am J Respir Crit Care Med 2014; 189: A6562.

21 Vestbo J, Hurd SS, Agustí AG, et al. Global strategy for the diagnosis, management, and prevention of chronic obstructive pulmonary disease: GOLD executive summary. Am J Respir Crit Care Med 2013; 187: 347-365.

22 National Emphysema Treatment Trial Research Group. Patients at high risk of death after lung-volume-reduction surgery. N Engl J Med 2001; 345: 1075-1083.

23 Szekely LA, Oelberg DA, Wright C, et al. Preoperative predictors of operative morbidity and mortality in COPD patients undergoing bilateral lung volume reduction surgery. Chest 1997; 111: 550-558.

24 Donohue JF. Minimal clinically important differences in COPD lung function. COPD 2005; 2: 111-124.

25 Gross NJ. Chronic obstructive pulmonary disease outcome measurements: what's important? What's useful? Proc Am Thorac Soc 2005; 2: 267-271.

26 Jones PW. St. George's Respiratory Questionnaire: MCID. COPD 2005; 2: 75-79.

27 Puhan MA, Chandra D, Mosenifar Z, et al. The minimal important difference of exercise tests in severe COPD. Eur Respir J 2011; 37: 784-790.

28 Kramer MR, Refaely Y, Maimon N, et al. Two-year follow-up in patients treated with emphysematous lung sealant for advanced emphysema. Chest 2013; 144: 1677-1680. 
29 Kramer MR, Fruchter O, Eberhardt R, et al. Emphysematous lung sealant therapy attenuates the chronic inflammatory state of advanced emphysema. J Pulm Respir Med 2013; 3: 163.

30 Herth FJ, Noppen M, Valipour A, et al. Efficacy predictors of lung volume reduction with Zephyr valves in a European cohort. Eur Respir J 2012; 39: 1334-1342.

31 Herth FJ, Eberhardt R, Gompelmann D, et al. Radiological and clinical outcomes of using Chartis ${ }^{\mathrm{TM}}$ to plan endobronchial valve treatment. Eur Respir J 2013; 41: 302-308.

32 Osaki S, Maloney JD, Meyer KC, et al. The impact of the lung allocation scoring system at the single national Veterans Affairs Hospital lung transplantation program. Eur J Cardiothorac Surg 2009; 36: 497-501. 